\title{
Clinical Considerations while increasing Occlusal Vertical Dimension
}

\author{
${ }^{1}$ Ravindra S Pawar, ${ }^{2}$ Rahul S Kulkarni, ${ }^{3}$ Sandeep K Pimpale
}

\begin{abstract}
A decrease in occlusal vertical dimension (OVD) may result from tooth structure loss and extraction of posterior teeth, and it affects the esthetics, function, comfort, and lower facial height (LFH). Rehabilitation of patients with worn dentitions may require increase in OVD and interarch distance to create space for restorative materials, improve esthetics, and develop physiologic occlusion. It is usually advisable to assess esthetics, function, $\mathrm{LFH}$, and response of the stomatognathic system to increased OVD by means of removable or fixed provisional appliances before definitive treatment. The following case report presents the multitude of factors considered in planning amount of OVD increase keeping in view its impact on LFH and facial esthetics, during rehabilitation of a patient with severely attrided dentition.
\end{abstract}

Keywords: Occlusal rehabilitation, Occlusal vertical dimension, Permissive occlusal splints, Provisional restorations.

How to cite this article: Pawar RS, Kulkarni RS, Pimpale SK. Clinical Considerations while increasing Occlusal Vertical Dimension. Int J Prosthodont Restor Dent 2017;7(3):103-107.

Source of support: Nil

Conflict of interest: None

\section{INTRODUCTION}

Occlusal vertical dimension has been defined as the distance measured between two selected anatomical or marked points when the occluding members are in contact, and in dentate individuals, OVD is maintained by occlusal contacts of teeth. ${ }^{1}$ A decrease in OVD may result from tooth structure loss and extraction of posterior teeth, and it affects LFH, function, comfort, and esthetics. Following tooth wear, an adaptive process continues in the alveolar bone to compensate for the loss of tooth structure and restore the OVD. Rehabilitation of patients with worn dentitions may require increase in OVD and interarch distance to create space for restorative materials,

\footnotetext{
${ }^{1,3}$ Assistant Professor, ${ }^{2}$ Associate Professor

${ }^{1,2}$ Department of Prosthodontics, Nair Hospital Dental College Mumbai, Maharashtra, India

${ }^{3}$ Department of Periodontics, Nair Hospital Dental College Mumbai, Maharashtra, India

Corresponding Author: Rahul S Kulkarni, Associate Professor Department of Prosthodontics, Nair Hospital Dental College Mumbai, Maharashtra, India, Phone: +912223082714, e-mail: drrahulprostho@yahoo.com
}

improve esthetics, and develop physiologic occlusion. ${ }^{2}$ Procedures involving the increase in OVD should be approached with caution, and a minimal increase in OVD should be applied, though a $5 \mathrm{~mm}$ maximum increase in OVD can be justified to provide adequate occlusal space for the restorative material and improve anterior teeth esthetics. Multiple techniques have been proposed to quantify OVD loss, such as pretreatment records, incisor height measurement, phonetic evaluation, assessment of facial appearance, cephalometric evaluation, and difference between VDR and OVD, to name a few. Although all the stated techniques have been found to be useful, none have been assessed to be scientifically more accurate than another, and it has been suggested that in order to improve the accuracy of the recording procedure, more than one method should be used. ${ }^{3}$ Following case report presents guidelines for planning OVD increase in patients, keeping in view its impact on LFH and facial esthetics. The patient described here had presented with severe attrition of tooth structure, multiple missing posterior teeth, and compensation of lost OVD through passive eruption of teeth.

\section{CASE REPORT}

\section{Case History and Intraoral Examination}

A 41-year-old woman presented for restorative treatment with the chief complaint of unesthetic appearance of her teeth, and also had difficulty during mastication and sensitivity to hot and cold. Careful intraoral examination revealed that she had severe attrition of teeth, and missing maxillary right first premolar and molar, mandibular right second, and left first molars, and mandibular right first premolar (Fig. 1). Discolored restorations were seen with maxillary anterior teeth. Periodontal examination indicated a healthy periodontium, and the interocclusal distance measured at the premolar region during physiological rest was $3 \mathrm{~mm}$. Esthetic evaluation revealed that patient had no facial asymmetry, had competent lips with decreased vermillion display, accentuated nasolabial and mentolabial folds, and decreased nose to chin distance $\mathrm{LFH}$. There were no signs or symptoms related to temporomandibular joint (TMJ), such as pain, limited range of jaw opening, or clicking. A panoramic radiograph revealed presence of multiple missing teeth, attrition of most teeth, and periapical pathologies with some teeth. 


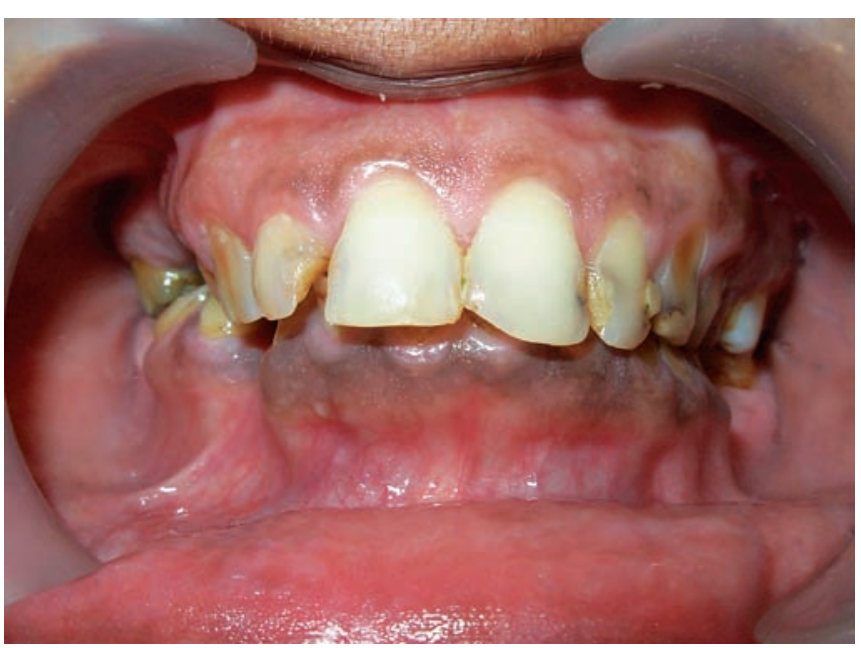

Fig. 1: Preoperative frontal view in centric occlusion. Note anterior deep bite and collapse of the arch due to loss of posterior teeth

Maxillary and mandibular complete arch diagnostic impressions were made using a heavy and light body vinylpolysiloxane impression material (Imprint; 3M ESPE, Seefeld, Germany). Two sets of diagnostic casts were made by pouring the impressions twice with a type III dental stone (Ultrastone, KalabhaiKarson, Mumbai, India). One cast set was used for the diagnostic wax-up, and the other was saved for patient records.

\section{Assessment of OVD and Interocclusal Rest Space, and Need to increase OVD}

Evaluation of existing OVD and interocclusal rest space (IORS) provides important initial reference in comprehensive treatment planning. The IORS, i.e., the difference in vertical dimension, when the mandible is at rest and in occlusion, was found to be $3 \mathrm{~mm}$ in the present case. An IORS of 2 to $3 \mathrm{~mm}$ has been suggested as the physiological space, which indicated that tooth eruption and alveolar bone growth compensated for the loss of OVD by attrition. ${ }^{2}$ The rationale behind measuring the IORS was to evaluate the need and feasibility of increasing the OVD. Phonetic evaluation was carried out by asking the patient to pronounce labiodentals fricatives, such as $\mathrm{f}$ and $\mathrm{v}$, and observation of Silverman's closest speaking space during production of sibilant sounds. Results of judging facial appearance by dividing face into three equal parts revealed that there was a decrease in $\mathrm{LFH}$ resulting from attrition of teeth. In essence, patient had an IORS of $3 \mathrm{~mm}$, while space requirement for PFM crowns would be a total of $3 \mathrm{~mm}$, i.e., an increase of $3 \mathrm{~mm}$ of OVD was required to develop esthetically pleasing restorations and satisfactory occlusion. A need to increase the OVD by $3 \mathrm{~mm}$ was justified after evaluating the space available for restorations, the remaining tooth structure, esthetic requirements, phonetics, and occlusion.

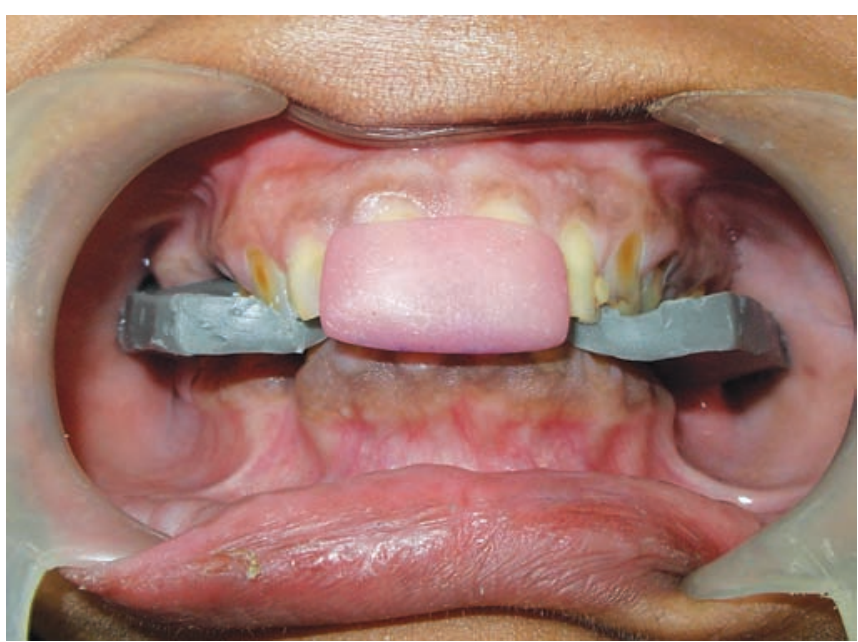

Fig. 2: Bite registration record for diagnostic mounting was made at $3 \mathrm{~mm}$ interincisal distance, using a Lucia jig and Aluwax

\section{Increase in OVD using Splint Therapy}

The maxillary cast was mounted on a semiadjustable articulator (Hanau H2, Whip Mix Corporation, Fort Collins, CO, USA) using a face-bow transfer. The mandibular cast was mounted using Lucia jig with $3.0 \mathrm{~mm}$ interocclusal distance in the anterior tooth region, and centric relation record (Fig. 2). Condylar angles were determined with the use of protrusive record, and the articulator was programmed. The permissive occlusal splint was fabricated with autopolymerizing acrylic resin, using sprinkle on method (Fig. 3). ${ }^{4,5}$ The splint was delivered to the patient to evaluate effect of increased OVD on LFH, TMJ, and masticatory muscles. Splint was designed to be retained on maxillary teeth, and had to be worn throughout except during meals and oral hygiene procedures. The patient was evaluated for 3 months to rule out presence of excessive signs of wear on the

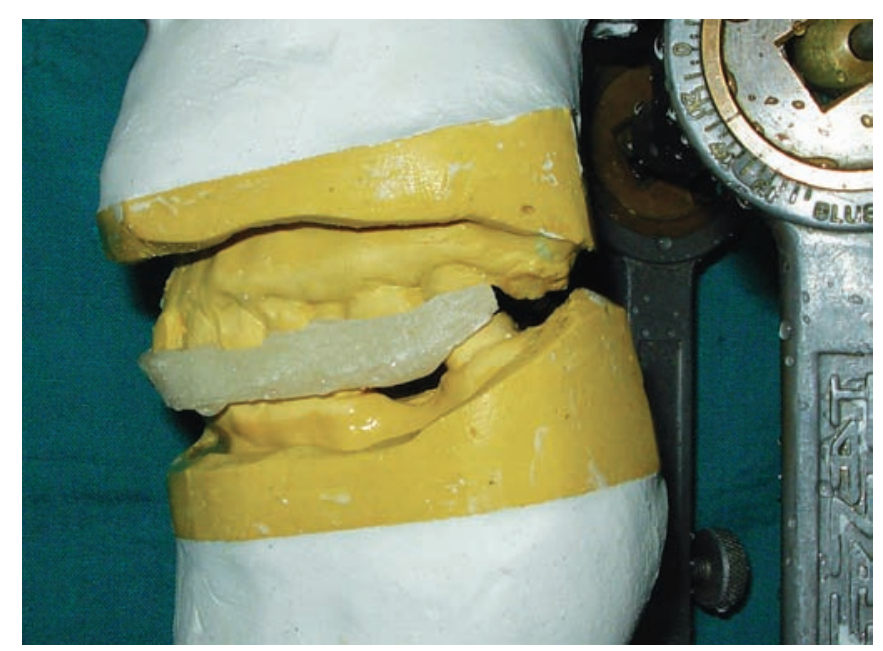

Fig. 3: Permissive occlusal splint at increased OVD. Occlusal indentations of mandibular teeth were removed during finishing and polishing to permit movements, while maintaining uniform contact of mandibular teeth in on splint in centric occlusion 


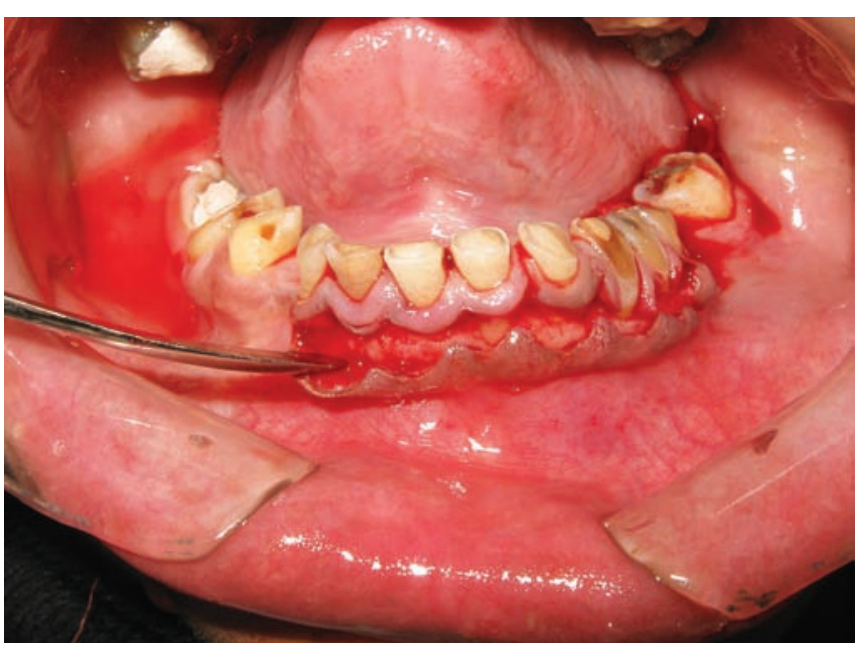

Fig. 4: The CLS was carried out in the mandibular arch to increase clinical heights of crowns and improve prognosis of the prostheses

appliance, symptoms of temporomandibular dysfunction, and muscle tenderness. The interocclusal space was evaluated at the end, and the definitive treatment was planned. The interocclusal space was observed to be $2 \mathrm{~mm}$, with splints in place, at the end of the assessment period.

The patient complained of sensitivity to hot and cold due to severe attrition of teeth, while few teeth also exhibited abrasion. Hence, intentional endodontic treatment of all teeth was carried out, to eliminate pulpal pathologies and also permit the operator to perform optimum amount of tooth reduction. Similarly, clinical crown lengthening surgery (CLS) with osteotomy was performed to provide adequate heights, and adequate resistance and retention forms in tooth preparations (Fig. 4).

\section{Provisional Fixed Dental Prostheses at Increased OVD}

Mounting of diagnostic casts was done at increased OVD, using anterior portion of splint modified as Lucia jig, and bite registration material. The diagnostic wax-up was developed at the increased OVD using the interocclusal space and golden proportion as guidelines, while mutually protected occlusal scheme was developed (Fig. 5). The provisional restorations were fabricated from acrylization of wax-up, which consisted of maxillary and mandibular full arch heat polymerized poly (methyl methacrylate) fixed dental prostheses (FDPs). Maxillary and mandibular teeth were prepared for porcelain fused to metal (PFM) restorations. ${ }^{6}$ The provisional FDPs obtained by acrylization of wax-up were internally relined using tooth colored autopolymerizing resin, and tried intraorally for occlusion, margins, and esthetics, and were cemented (Fig. 6). Periodic recalls were scheduled for 3 months. To record and preserve the anterior guidance of the provisional restorations, a custom incisal guide table was fabricated from acrylic resin (Pattern Resin LS; GC America).

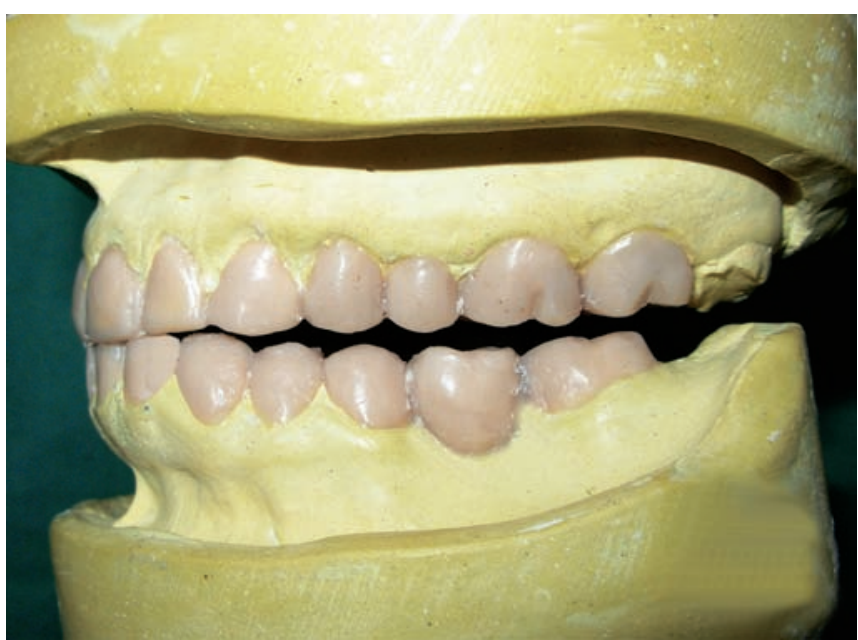

Fig. 5: Diagnostic wax-up was developed using mutually protected occlusal scheme. Disclusion of posterior teeth by anteriors has been depicted here

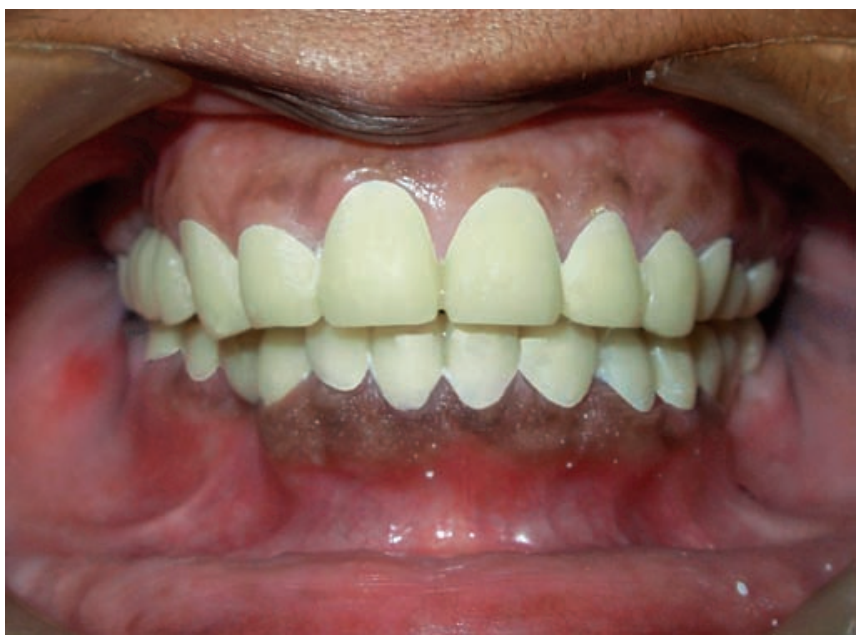

Fig. 6: Maxillary and mandibular complete arch fixed provisional restorations, at increased OVD, in place

\section{Evaluation of Patient at Increased OVD}

As described earlier, patient's OVD was increased using splint therapy and provisional FDPs, and periodic recalls were carried out for 3 months to evaluate effect of the altered OVD on stomatognathic system. To perform this, following criteria described by Bloom were applied, ${ }^{7}$ and presented in Table 1.

\section{Fabrication of Definitive Restorations}

Final tooth modification and gingival retraction were carried out in the maxillary and mandibular arches, and definitive impressions were recorded using polyvinyl siloxane impression material (Affinis; Coltène/ WhaledentInc, Cuyahoga Falls, Ohio). Definitive casts were obtained using die stone, and bite registration was taken using provisional crowns and occlusal registration material (StoneBite; DreveDentamid $\mathrm{GmbH}$, Unna, Germany) in sections. Casts were transferred to 
Table 1: Effect of the altered OVD on stomatognathic system

\begin{tabular}{|c|c|c|}
\hline Test & Result & Inference \\
\hline Load test & Negative & $\begin{array}{l}\text { No sign of tension or tenderness was } \\
\text { observed in TMJs at vertical loading }\end{array}$ \\
\hline Clench test & Negative & $\begin{array}{l}\text { No sign of tension or tenderness in } \\
\text { TMJs or teeth under occlusal loading }\end{array}$ \\
\hline Grinding test & Negative & No posterior interferences \\
\hline Fremitus test & Negative & Stable occlusal contacts \\
\hline Comfort test & Positive & $\begin{array}{l}\text { Complete comfort of lips, face, and } \\
\text { teeth with no speech problems }\end{array}$ \\
\hline Esthetic test & Positive & $\begin{array}{l}\text { Patient and dentist completely } \\
\text { satisfied with the facial appearance }\end{array}$ \\
\hline Stability test & Positive & $\begin{array}{l}\text { No sign of instability, i.e., wear or } \\
\text { dislodgement of restorations }\end{array}$ \\
\hline
\end{tabular}

the semiadjustable articulator using facebow and bite registrations. Individual PFM (SuperCast, Talladium Inc., Valencia, USA; IPS Classic, IvoclarVivadent AG, Schaan, Liechtenstein) crowns and FDPs were made using customized anterior guide table fabricated previously. The prostheses were designed using mutually protected occlusion in which the anterior teeth protected the posterior teeth from excursive force and wear, and posterior teeth supported the bite force. During bisque trial, centric occlusion, anterior guidance, and posterior disclusion were verified in the definitive restorations. Long centric occlusion was developed in the maxillary anterior restoration to allow for freedom in anterior posterior movement. This was followed by glazing of the ceramo-metal crowns, and finally their cementation with phosphate cement (SuperCement, Shofu, Kyoto, Japan) (Fig. 7). Oral hygiene instructions were given and brushing technique was demonstrated. Recall evaluations were carried out at 3-month intervals for a period of 1 year. The patient's esthetic and functional expectations were satisfied, and she did not have sensitivity or pain after the treatment (Fig. 8).

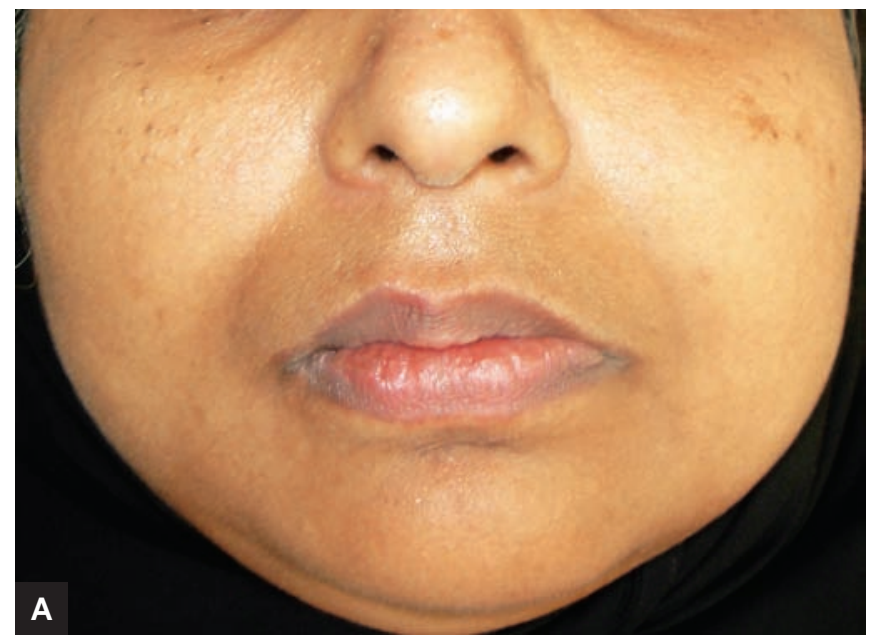

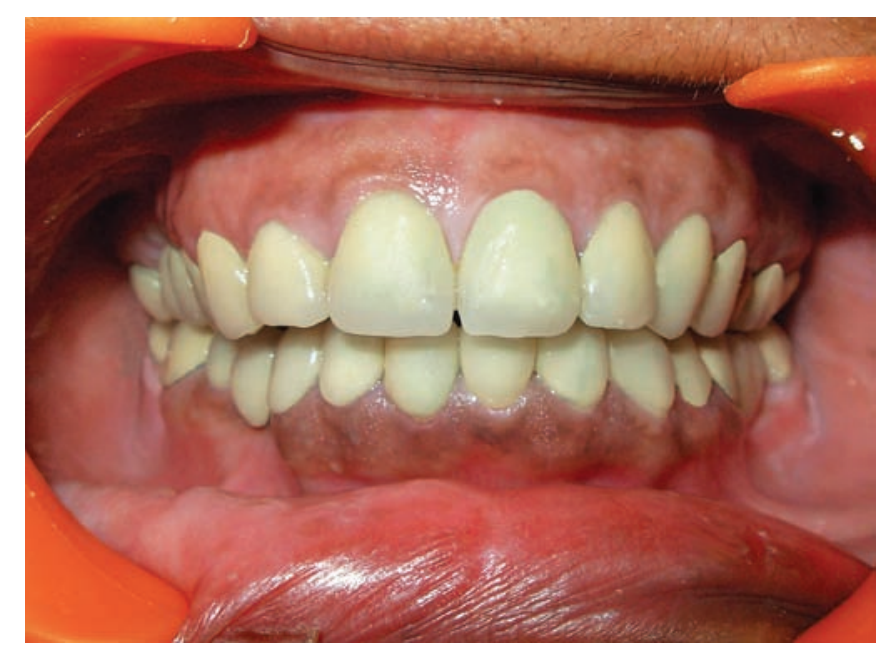

Fig. 7: Maxillary and mandibular complete arch fixed final restorations in place

\section{DISCUSSION}

Alteration of VDO by increasing the interarch distance is frequently performed in oral rehabilitation to enhance esthetic tooth display, improve lip support, establish anatomic harmony, and improve phonetics. ${ }^{2}$ Increasing the OVD from clinical perspective has been reported to facilitate the treatment of patients presenting with generalized and complex dental abnormalities, such as generalized tooth wear and significant occlusal irregularities. Proper occlusal adjustment with occlusal devices will reduce muscle tenderness and TMD symptoms. ${ }^{3}$ In order to achieve a predictable and desirable prognosis, a systematic approach may be helpful. A patient's adaptation to the new resting posture is controlled by neuroreceptors located in the periodontal ligament, the masticatory mucosa, and TMJ.

Tooth wear may be associated with loss of OVD, poor esthetics, difficulty during mastication, tooth sensitivity, and compensation of the lost OVD by gradual eruption of

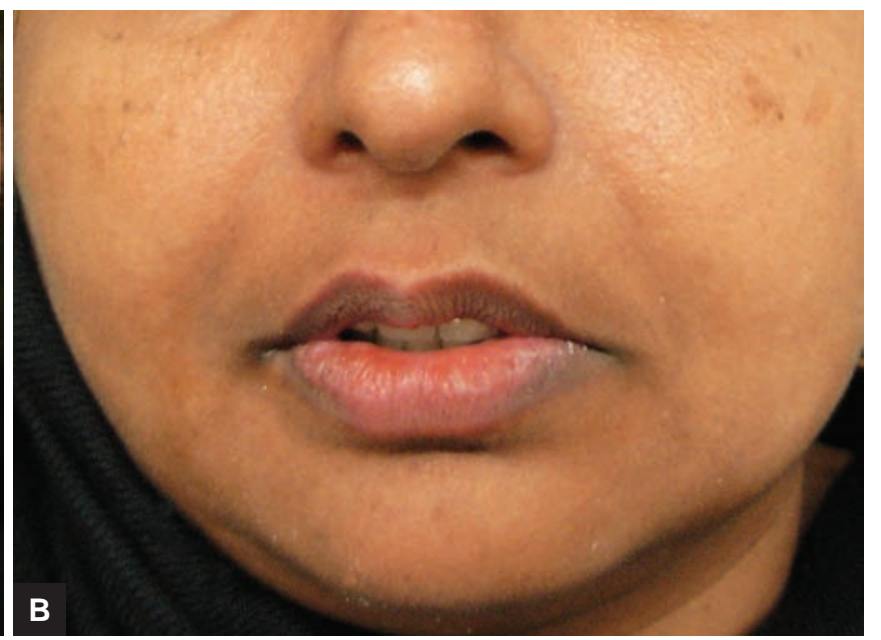

Figs 8A and B: (A) Pretreatment extraoral photograph. Note prominent nasolabial and mentolabial folds, inconspicuous vermillion border, and decreased LFH; (B) Posttreatment extraoral photograph at 1 year recall interval indicates restoration of OVD and lower facial esthetics 
teeth. It is usually advisable to assess esthetics, function, and response of the stomatognathic system to increase in OVD by means of removable (e.g., removable overlay splints, interim partial dentures, occlusal splints, etc.) or fixed (e.g., base metal onlays, fixed provisional restorations, and composite resin restorations) provisional appliances before definitive treatment. ${ }^{4}$ Both entities have their advantages and disadvantages, for instance, patient compliance is an issue with removable splints, while irreversible tooth preparation is required with fixed provisionals. It is plausible to evaluate LFH, function, and OVD with permissive occlusal splints, before invasive procedures like tooth preparation and surgical crown lengthening are performed, when adequate patient compliance is present. ${ }^{2}$ Hence, permissive occlusal splints were fabricated for the patient to evaluate effect of increasing the OVD on LFH and function. An increase of $3 \mathrm{~mm}$ in OVD was planned in the said patient, while an increase of up to $5 \mathrm{~mm}$ interincisally has been considered as feasible alteration. ${ }^{8}$ Even though there are no clear objective guidelines to determine the ideal increase of the OVD, there is neither strong evidence supporting the pathologic effects of the same, and most of the studies agreed that patient adaptation can be obtained after increasing the OVD. ${ }^{2}$ The present case had a generalized loss of crown height due to tooth wear, and it was considered desirable to increase OVD initially with noninvasive treatment like permissive occlusal splint. Once patient's response to increased OVD was found to be favorable, provisional fixed bridges were provided. An advantage of this approach is that, invasive procedure of tooth preparation was deferred until patient was comfortably adjusted to the increased OVD. ${ }^{3}$

\section{CONCLUSION}

This clinical report describes a systematic approach to increase OVD in a patient with generalized attrition. It is advisable to assess esthetics, function, and response of the stomatognathic system to increase in OVD by means of removable appliances initially, and later by fixed provisional appliances before definitive treatment. The CLS and endodontic treatment may be carried out before or during fixed provisional restoration stage. Various clinical tests may be conducted to evaluate favorable response of patient to increased OVD, and definitive restorations, such as ceramometal crowns and FDPs can be delivered.

\section{REFERENCES}

1. The glossary of prosthodontic terms. J Prosthet Dent 2005 Jul;94(1):10-92.

2. Abduo J. Safety of increasing vertical dimension of occlusion: a systematic review. Quintessence Int 2012 May;43(5):369-380.

3. Ormianer Z, Palty A. Altered vertical dimension of occlusion: a comparative retrospective pilot study of tooth-and implantsupported restorations. Int J Oral Maxillofac Implants 2009 May-Jun;24(3):497-501.

4. Dawson, PE. Functional occlusion: from TMJ to smile design. 1st ed. St. Louis (MO): Mosby-Elsevier; 2007. p. 150-158, 379-392.

5. Phoenix, RD. Prosthetic polymers and resins. In: Anusavice KJ, editor. Phillips' science of dental materials. 12th ed. Philadelphia (PA): Saunders-Elsevier; 2012. p. 474-498.

6. Rosensteil, SF. Principles of tooth preparation. In: Rosensteil S, Land M, Fujimoto J, editors. Contemporary fixed prosthodontics. 4th ed. St Louis (MO): Mosby-Elsevier; 2006. p. 209-258.

7. Bloom DR, Padayachy JN. Increasing occlusal vertical dimension-why, when and how. Br Dent J 2006 Mar;200(5):251-256

8. Yabushita T, Zeredo JL, Toda K, Soma K. Role of occlusal vertical dimension in spindle function. J Den Res 2005 Mar;84(3):245-249. 